\title{
IMPLICACIÓN DEL CONTEXTO SOCIOECONÓMICO EN LA EXPOSICIÓN A PLAGUICIDAS EN JORNALEROS HUICHOLES
}

\author{
Gilberto LÓPEZ-MARTÍNEZ ${ }^{1}$, Diana Marcela PAREDES-CÉSPEDES ${ }^{1}$, \\ Aurora Elizabeth ROJAS-GARCÍA ${ }^{2}$, Irma Martha MEDINA-DÍAZ ${ }^{2}$, \\ Briscia Socorro BARRÓN-VIVANCO², Cyndia Azucena GONZÁLEZ-ARIAS ${ }^{2}$ \\ y Yael Yvette BERNAL-HERNÁNDEZ ${ }^{2} *$
}

${ }^{1}$ Posgrado en Ciencias Biológico Agropecuarias, Unidad Académica de Agricultura. Km. 9 Carretera Tepic Compostela. Xalisco, Nayarit, México

${ }^{2}$ Laboratorio de Contaminación y Toxicología Ambiental. Secretaría de Investigación y Posgrado. Universidad Autónoma de Nayarit. 63155. Ciudad de la Cultura s/n. Col. Los Fresnos, C.P. 63190. Tepic, Nayarit, México *Autor para correspondencia: yael.yvette@gmail.com

(Recibido enero 2017; aceptado septiembre 2017)

Palabras clave: Indígenas, migración, plaguicidas

\section{RESUMEN}

Los huicholes se han dedicado a través de la historia, al trabajo de la tierra para subsistir, esta actividad juega un papel esencial dentro de su cosmovisión. Como medida de subsistencia, el pueblo huichol ha recurrido a la migración a diferentes regiones de la costa del estado de Nayarit, donde se emplean en trabajos relacionados principalmente con la agricultura y la aplicación de plaguicidas. Se ha observado que, en estas poblaciones, los efectos de los plaguicidas en la salud pudieran verse aumentados por diferentes factores socioeconómicos y culturales que deben ser considerados para su análisis. Este estudio, es un acercamiento exploratorio para conocer los diferentes aspectos socioeconómicos y culturales implicados en el uso y manejo de plaguicidas en dos comunidades indígenas del estado de Nayarit. En esta investigación, se observó que los plaguicidas más utilizados son los organofosforados (POF). El 90\% de la población realiza actividades de siembra, cosecha, corte y ensarte de tabaco, además de aplicar y mezclar plaguicidas. Así también, el $74 \%$ de los trabajadores desconoce que compuestos utiliza y el $26 \%$ utiliza mezclas de éstos. Este trabajo, contribuirá al desarrollo de propuestas que mejoren las condiciones laborales de los jornaleros indígenas de la región.

Keywords: Indigenous, migration, pesticides

\begin{abstract}
Huichols have dedicated historically to work the land in order to subsist, which is an activity that plays an essential role in their culture. To subsist, Huichols are forced to migrate to different regions in the coast of Nayarit state, where they employ in agricultural works such as application of pesticides. Due to different socio-economic and cultural factors, the potential detrimental effects triggered by pesticides exposure in Huichols might be exacerbated, deserving attention. Hence, this article is an exploratory approach of the socioeconomic and cultural aspects involved in the use and management of pesticides in two indigenous communities in the state of Nayarit. The data gathered showed that organophosphates (OP) are the most used compounds. Also, $90 \%$
\end{abstract}


of the population works in activities including sowing, harvesting, cutting and stringing tobacco, as well as in applying and mixing pesticides. Further, $74 \%$ workers does not have knowledge of the type of pesticides they handle, and $26 \%$ uses more than one pesticide at a time. This study may contribute to develop proposals aimed to improve the working conditions of indigenous farmworkers in the studied region.

\section{INTRODUCCIÓN}

Los plaguicidas son compuestos asociados con la agricultura intensiva, así como con el control de plagas y de vectores trasmisores de enfermedades (FAO 2003). La producción, uso y exposición a estos compuestos tóxicos en el ambiente, impacta la salud, bienestar, cultura, alimentación y seguridad de los pueblos indígenas, y por ende los derechos individuales de esta población. De 80,000 productos comerciales, más del $85 \%$ no han sido evaluados sobre sus efectos a la salud humana y mucho menos en poblaciones vulnerables como las comunidades indígenas (Carmen y Waghiyi 2012).

Los pueblos indígenas de México viven en zonas desérticas o montañosas y subsisten principalmente de la tierra a través de la agricultura, pastoreo, caza y recolección de alimentos. Muchas de estas regiones tienen una exposición importante a contaminantes tóxicos como resultado de la alta actividad de industrias extractivas de minerales, gas y petróleo; así como por la agricultura que depende en gran medida de un alto uso de plaguicidas (Carmen y Waghiyi 2012).

\section{Pueblos indígenas de México y el uso de plagui- cidas}

En México las poblaciones indígenas conforman un grupo de pueblos originarios que, dada su cultura, historia y dialecto, dan sentido de pertenencia e identidad al país. Estas poblaciones están ubicadas dentro de la sociedad, como un grupo con una identidad social y cultural propia, con un gran apego al territorio ancestral, y una producción orientada principalmente a la subsistencia (INEE 2006). Navarrete (2008) los define como los descendientes de las poblaciones que habitan en el territorio mexicano desde antes de la llegada de los españoles, aclara también que son, únicamente aquellos que conservan total o parcialmente la cultura, instituciones y forma de vida de estos pueblos prehispánicos.

La actividad económica en estas poblaciones de México muestra un panorama muy diverso. Sin embargo, se ha registrado que aproximadamente el $52.4 \%$ de la población indígena se dedica a la agricultura, mientras el 19.6 y $28 \%$ se dedican al sector secundario y terciario respectivamente (CDI 2000).

De acuerdo con la encuesta intercensal realizada en el año 2015, en el estado de Nayarit la etnia que se encuentra en mayor proporción es la huichol, seguida de coras, tepehuanos y mexicaneros (INEGI 2016). El pueblo Wixárika (huichol) abarca porciones de cuatro estados: Jalisco, Nayarit, Durango y Zacatecas (Neurath 2003). Esta población se asienta de manera dispersa en el territorio, mientras en Jalisco los huicholes han logrado impedir el asentamiento de mestizos al interior de sus comunidades, en Nayarit es frecuente la convivencia en el mismo territorio con coras o mestizos. Son cinco los centros ceremoniales del pueblo Wixárika en donde se instalan los gobiernos tradicionales: San Andrés Cohamiata (Tateikie), Santa Catarina Cuexcomitlán (Tuapurie), San Sebastián Teponahuaxtlán (Wuatia) y Tuxpan de Bolaños (Tutsipa) en el estado de Jalisco y Guadalupe Ocotán (Xatsitsarie) en Nayarit (Ochoa 2001, Álvarez 2015).

Los huicholes se han dedicado a través de la historia, al trabajo de la tierra para subsistir. Además, esta actividad juega un papel esencial dentro de su cosmovisión (Neurath 2003). Como medida de sostén, el pueblo huichol ha migrado a diferentes regiones de la costa del estado de Nayarit, donde se emplea principalmente en trabajos relacionados con la agricultura y la aplicación de plaguicidas (Díaz y Salinas 2002). En esta actividad, se emplean mujeres, hombres y niños como mano de obra, mismos que no utilizan equipo de protección adecuado y por consecuencia son vulnerables a los efectos tóxicos de estos productos (Díaz y Salinas 1998, 2002, Ramos 2012, Gamlin 2013). Además, las principales características del pueblo huichol, sus costumbres, creencias y condiciones de vida, pudieran impactar de manera importante sobre la exposición a plaguicidas, especialmente en los trabajadores agrícolas huicholes.

En México, son escasas las investigaciones realizadas en este grupo vulnerable debido a la dificultad que representa trabajar en poblaciones con un dialecto y costumbres definidas; así como, por su alta tasa de migración durante diferentes épocas del año y al accidentado acceso a sus comunidades de origen. 


\section{Huicholes y la migración a los campos agrícolas en Nayarit}

La agricultura es una actividad económica importante en Nayarit, por consecuencia, la demanda de mano de obra provoca un fenómeno migratorio de jornaleros huicholes a los campos agrícolas del estado. En sus comunidades de origen los jornaleros trabajan sus tierras para el autoconsumo y en época de sequía (enero-mayo), cuando sus tierras son improductivas, migran a la planicie costera para emplearse en la cosecha y cultivo de diversos productos tales como: tabaco, frijol, chile y tomate, entre otros (Mackinlay 2008). Cada año, aproximadamente el $40 \%$ de las familias huicholas dejan sus comunidades para buscar empleo en los campos agrícolas, donde se emplean en diversas actividades que incluyen preparación de tierra para cultivo, cosecha de diversos productos y aplicación de plaguicidas. Durante su estancia en la planicie costera, los jornaleros huicholes viven dentro de las plantaciones, cocinan sus alimentos al ras del suelo y con frecuencia beben agua proveniente de canales de riego, arroyos y pozos (Díaz y Salinas 2002).

La migración de los indígenas, obedece principalmente a la necesidad de contar con un ingreso que permita la supervivencia, en muchas ocasiones el jefe de familia migra a los campos acompañado de su familia completa, lo que implica también la exposición de niños y mujeres a los riesgos que conlleva el establecerse en el campo donde se vierten plaguicidas potencialmente tóxicos (Pacheco 1999, Gamlin 2016).

Intoxicación con plaguicidas un problema cultural, económico y social en las comunidades indígenas huicholas

Los huicholes son agricultores expertos que durante siglos se han mantenido principalmente con la producción de maíz. De acuerdo con su cosmovisión, solo aquellos que pueden hacer crecer variedades sagradas de maíz pueden participar de las ceremonias; y solo aquellos que participan de las ceremonias pueden por tradición, trabajar la tierra (Neurath 2003).

De acuerdo con el Sistema Nacional de Vigilancia Epidemiológica en 2009, Nayarit presentó los niveles más altos de intoxicación por plaguicidas en México. Asimismo, se ha reportado que las mujeres que trabajan en el campo tienen 3.61 veces más probabilidad de tener hijos con malformaciones, en comparación con las mujeres que no trabajan en el sector agrícola (Gamlin 2013). Sin embargo, los datos médicos son muy escasos y existe carencia de información epidemiológica o datos concernientes a comunidades huicholas.

Son escasos los estudios en la literatura científica en los que se evalúan biomarcadores toxicológicos relacionados con plaguicidas en poblaciones huicholas. Gamlin et al. (2006), evaluaron la actividad acetilcolinesterasa (AChE) como biomarcador de exposición en niños jornaleros huicholes en los campos tabacaleros de Nayarit. Asimismo, BernalHernández et al. (2014) evaluaron una batería de biomarcadores toxicológicos en jornaleros indígenas huicholes del estado de Nayarit. El presente trabajo tiene por objetivo analizar la implicación del contexto socioeconómico en la exposición a estos compuestos en jornaleros huicholes.

\section{MATERIALES Y MÉTODOS}

\section{Diseño del estudio}

Este trabajo se realizó en dos comunidades del estado de Nayarit: Santiago de Pochotitán y Jesús María Corte, ambas comunidades huicholas dedicadas principalmente a la agricultura. Durante la temporada de enero a mayo, migran a la región costera para trabajar en los campos. Una vez finalizada esta temporada, regresan a su comunidad de origen y se dedican a la preparación de sus tierras para cultivo.

Se realizó una visita a cada comunidad para invitar a los participantes y dar a conocer los objetivos del estudio. Se consideraron como criterios de inclusión: que fueran jornaleros huicholes, mayores de 18 años y que participaran de manera voluntaria en el estudio. Asimismo, debían cumplir con los criterios establecidos por la Comisión Nacional para el Desarrollo de los Pueblos Indígenas (CDI): portar el grupo sanguíneo $\mathrm{O}$ (sistema $\mathrm{ABO}, \mathrm{Rh}+$ ), compartir tradiciones culturales propias de la etnia y hablar el dialecto huichol. En este estudio participaron 126 jornaleros huicholes, mismos que firmaron una carta de consentimiento informado y posteriormente se les pidió una muestra de sangre para las determinaciones analíticas. El estudio fue aprobado por la Comisión de Bioética de Nayarit (CEBN/01 /2016).

Para conocer el fenómeno plaguicidas-población, se aplicó una entrevista exploratoria y descriptiva. La recolección de datos se hizo a partir de un cuestionario estructurado que se aplicó a cada jornalero para conocer las características socioeconómicas, antropométricas, edad, dieta, hábitos nocivos (consumo de drogas, tabaco y alcohol), exposición, así como síntomas relacionados con ésta y el uso adecuado de equipo de protección. 


\section{Recolección y procesamiento de muestras}

Como biomarcadores de exposición se midió la actividad acetilcolinesterasa (AChE) y butirilcolinesterasa (BuChE). A los participantes se les tomaron muestras de sangre venosa, la cual fue extraída por venopunción y colectada en tubos BD Vacutainer ${ }^{\circledR}$ sin anticoagulante y con EDTA como anticoagulante.

\section{Determinación de la actividad acetilcolinesterasa (AChE)}

La actividad AChE se midió de acuerdo al método propuesto por Ellman et al. (1961). Se realizó una dilución 1:100 de las muestras de sangre con el detergente tritón X-100 (0.03\%). Se mezclaron $0.5 \mathrm{~mL}$ de la dilución con $1 \mathrm{~mL}$ de la solución amortiguadora de fosfatos $(0.1 \mathrm{M}, \mathrm{pH} 7.4), 0.05 \mathrm{~mL}$ del ácido 2,2-dinitro-5,5-ditiobenzoico (DNTB) $(10 \mathrm{mM})$ y $0.005 \mathrm{~mL}$ de etopropazina $(6 \mathrm{mM})$. Posteriormente, la mezcla se incubó a $37^{\circ} \mathrm{C}$ durante 10 minutos y se agregaron $0.025 \mathrm{~mL}$ de yoduro de acetiltiocolina $(28.3 \mathrm{mM})$. A continuación, se registró el cambio de absorbancia a $436 \mathrm{~nm}$ durante 3 minutos. Las mediciones se realizaron en un espectrofotómetro Spectronic Genesys 10Bio (Wisconsin, USA). La actividad AChE fue corregida por el contenido de hemoglobina y se expresó como U/g de Hb.

\section{Determinación de hemoglobina}

La concentración de Hb se determinó a través del método de Drabkin, a partir de $10 \mu \mathrm{L}$ de sangre total que se diluyeron en $2.5 \mathrm{~mL}$ de reactivo de Drabkin. Posteriormente se incubó 10 minutos en oscuridad a temperatura ambiente y se determinó su absorbancia a $540 \mathrm{~nm}$.

\section{Determinación de la actividad butirilcolinesterasa (BuChE)}

La actividad BuChE se determinó utilizando el kit "Colinesterasa (CHE) butirilcolina" de Biosystems ${ }^{\circledR}$ de acuerdo con las especificaciones del fabricante. Para preparar el reactivo de trabajo se colocaron en un tubo $1200 \mu \mathrm{L}$ del reactivo A con $300 \mu \mathrm{L}$ de reactivo $\mathrm{B}$ y se precalentó a $37^{\circ} \mathrm{C}$ durante 10 minutos. Posteriormente, se agregaron $25 \mu \mathrm{L}$ de suero, se mezcló y se midió en un espectrofotómetro Spectronic Genesys 10Bio (Wisconsin, USA) a $405 \mathrm{~nm}$ cada 30 segundos, durante 4 minutos. La actividad catalítica se reportó en U/L.

\section{Análisis estadístico}

Para evaluar la distribución de los datos se aplicó la prueba de Kolmogorov- Smirnov. Cuando los datos sugirieron una distribución normal se aplicó un análisis de varianza (ANOVA), y la prueba de Kruskal-Wallis para comparar más de dos grupos cuando los datos no tuvieron una distribución normal. Valores de $p<0.05$ se consideraron estadísticamente significativos. Para el análisis estadístico se utilizó el paquete estadístico STATA versión 8.0 (Stata Corp LP, College Station, Texas, USA).

\section{RESULTADOS Y DISCUSIÓN}

La recolección de datos a través de cuestionario se hizo con apoyo del juez de cada comunidad, quien facilitó el intercambio de información entre el entrevistado y el encuestador. Se tuvo una tasa de respuesta del $80 \%$. El $56 \%$ de los participantes fueron mujeres y $44 \%$ hombres, con una media geométrica de 35.7 (18-80) años de edad (Cuadro I).

Los huicholes son de las etnias con mayor presencia en el estado de Nayarit, debido al aislamiento en que han vivido, son de los grupos indígenas genética, cultural y tradicionalmente más conservados en México (Neurath 2003). Las ceremonias más importantes de los huicholes están estrechamente relacionadas con el ciclo agrícola donde está implícito el uso de plaguicidas. De acuerdo con lo reportado, en la temporada de sequía migran a la costa del estado donde se emplean en actividades agrícolas. Al respecto, se observó que el $90 \%$ de los participantes realizan actividades de siembra, cosecha, corte y ensarte de tabaco, además de aplicar y mezclar plaguicidas. Asimismo, se identificó que en promedio trabajan 9.2 horas al día con un rango de 1 a 16 horas y 6 días a la semana (Bernal-Hernández et al. 2014).

Según datos del INEGI (2010) Nayarit ocupa el séptimo lugar en relación a la tasa de analfabetismo a nivel nacional; el $30 \%$ de la población indígena del estado es considerada analfabeta, particularmente en los municipios del Nayar, Huajicori y Santa María del Oro.

Respecto al uso de plaguicidas, se observó que el $74 \%$ de los trabajadores desconoce los productos que utiliza y el $26 \%$ utiliza más de un compuesto al mismo tiempo en forma de mezclas. Los más utilizados por la población de estudio fueron los organofosforados (POF): azinfos-metil, clorpirifos-etil, diazinón, malatión, metilparatión y terbufos (Cuadro II), el $33.33 \%$ de éstos son clasificados dentro del grupo IA, es decir extremadamente peligrosos de acuerdo con la clasificación toxicológica de la organización mundial de la salud (OMS). Así también, se identificó que el $90 \%$ de los trabajadores no reciben capacitación ni son informados acerca del uso y de los efectos 
CUADRO I. CARACTERÍSTICAS ANTROPOMÉTRICAS DE LA POBLACIÓN DE ESTUDIO

\begin{tabular}{lccc}
\hline Características & Jesús María Corte & Santiago de Pochotitán & Valor de $\mathrm{p}$ \\
\hline Sexo (\%) & & & \\
$\quad$ Mujeres & 56.45 & 57.81 & 0.87 \\
$\quad$ Hombres & 43.55 & 42.19 & \\
Edad (\%) & & & \\
$\quad 18-24$ & 20.96 & 20.31 & 0.86 \\
$25-34$ & 30.64 & 26.56 & \\
$35-49$ & 27.41 & 34.37 & \\
$\geq 50$ & 20.96 & 18.75 & \\
IMC (\%) & & & \\
$\quad$ Normal & 48.08 & 48 & 0.085 \\
$\quad$ Sobrepeso & 36.54 & 52 & 0.13 \\
$\quad$ Obesidad & 15.38 & - & \\
$\quad$ Hábito de fumar (\%) & 12 & - & \\
\hline
\end{tabular}

IMC: Normal=18.50-24.99 $(\mathrm{Kg} / \mathrm{m} 2)$, sobrepeso= 25.00-29.99 $(\mathrm{Kg} / \mathrm{m} 2)$, obesidad $=\geq 30$

$\left(\mathrm{Kg} / \mathrm{m}^{2}\right)$. El valor de $\mathrm{p}$ se obtuvo con la prueba de ji-cuadrada

CUADRO II. PLAGUICIDAS MÁS UTILIZADOS POR LAS COMUNIDADES DE ESTUDIO

\begin{tabular}{lllc}
\hline Plaguicida & Clase química & Nombre común & $\begin{array}{c}\text { Clasificación } \\
\text { toxicológica }\end{array}$ \\
\hline \multirow{3}{*}{ Insecticida } & Organofosforado & Azinfos-metil & IB \\
& & Chlorpirifos etil & II \\
& & Diazinón & II \\
& & Malatión & III \\
& & Metil paratión & IA \\
\hline \multirow{3}{*}{ Herbicida } & Terbufos & IA \\
& Carbamato & Lannate 90 & IB \\
& Ácido-diclorofenoxiacético & Esteron & III \\
& (2-4-D) & Glifosato & III \\
& N-fosfonometilglicina & Dicloruro de Paraquat & IA \\
\hline
\end{tabular}

Clasificación toxicológica de la OMS (2009): Clase IA-extremadamente peligroso, clase IB-altamente peligroso, clase II-moderadamente tóxico, clase III-ligeramente tóxico, clase U-prácticamente no tóxico

de los agentes que utilizan. Bernal-Hernández et al. (2014) refirieron que los jornaleros huicholes tienen un mayor riesgo de exposición debido a diversos factores, entre ellos el analfabetismo, que dificulta el entendimiento de las instrucciones sobre el uso y manejo de estos compuestos y a las condiciones en que viven y laboran dentro de los campos agrícolas. Asimismo, Pacheco (1999) sustenta que aunado al analfabetismo, el monolingüismo es un factor que aumenta el riesgo de exposición a plaguicidas en estas comunidades, en algunos casos el conocimiento del español es muy limitado o nulo, al ser el dialecto wixárika la lengua materna.
Un estudio realizado, por Salinas y Díaz (2000) menciona la dificultad de los huicholes migrantes para leer la información que está escrita en los envases de los plaguicidas. Además, explica que en la cultura wixárika el cráneo con los huesos cruzados presente en estos contenedores, no siempre se relaciona con peligro, ya que el símbolo de calaveras tiene un significado diferente para esta etnia, asociado especialmente a la relación con sus dioses.

Por otra parte, el $50 \%$ de los trabajadores indicaron que nunca habían usado equipo de protección durante la jornada laboral. En este sentido Pacheco (1999) indicó que la capacitación en las actividades 
agrícolas se lleva a cabo por los mismos integrantes de la familia, debido a la falta de compromiso por parte de los contratistas al no asumir los costos para la capacitación o por desconocimiento propio.

De acuerdo con lo reportado por los jornaleros, éstos presentan una historia ocupacional de tipo crónica, dado que el $40 \%$ de la población de estudio refirió tener 15 años de trabajar en el campo e incluso algunos más de 50 años. Aunado al tiempo de exposición, los jornaleros viven y laboran en los campos agrícolas, una vez contratados construyen sus viviendas temporales dentro de los campos de cultivo, la mayoría de éstas son de una sola habitación la cual sirve de dormitorio, cocina, almacén de granos y utilería para la agricultura, estas condiciones favorecen la presencia de sintomatología asociada con la exposición a estos compuestos (Pacheco 1999). Al respecto, al preguntar si consideraban el uso de éstos como peligroso, el $90 \%$ de los participantes respondieron que sí, y el $82.3 \%$ refirieron tener dolor de cabeza, 54\% informó tener sensación de vómito y el $75 \%$ mareos durante la jornada laboral. Asimismo, el $68 \%$ de la población indicó ardor en los ojos, $69.1 \%$ dolor de estómago y $40.9 \%$ salivación excesiva. Resultados similares fueron reportados por Neupane et al. (2014) en una población de agricultores de Nepal expuestos a POF y carbamatos, quienes refirieron síntomas como: cansancio extremo (47\%), visión borrosa $(50 \%)$, sudoración excesiva $(43 \%)$, dolor de cabeza $(40 \%)$ y calambres musculares $(40 \%)$.

Atender los síntomas inducidos por la exposición a plaguicidas se dificulta en estas poblaciones, sobre todo cuando la sintomatología se relaciona más con sus creencias. De acuerdo con su cosmovisión, éstos se deben a un castigo divino debido al incumplimiento de las costumbres o al desacuerdo con alguna conducta wixárika, que se resuelve con ayuda del marakame (Pacheco 1999).

La OMS (1999) afirmó en la declaración sobre salud y supervivencia de los pueblos indígenas, que el concepto de salud en estas comunidades incluye una perspectiva holística que incorpora cuatro dimensiones de la vida: espiritual, intelectual, física y emocional. Lo anterior resulta importante de considerar cuando se trabaja con poblaciones como la etnia huichol, debido a que la espiritualidad continúa siendo un aspecto central que rige su vida y el orden social (Koenig 2007). Específicamente, en los huicholes existe una estrecha relación entre la espiritualidad y cultura con el ambiente (Eckersley 2007), los cuales son aspectos claves en su identidad, y fundamentales en su relación con la migración agrícola y por lo tanto con el uso de plaguicidas.
Por otro lado, los POF pueden inhibir la actividad de las colinesterasas en diferente grado (De la Iglesia y Delgado 2000, Costa et al. 2005). Diversos estudios en poblaciones han reportado una disminución en la actividad de las colinesterasas (Hernández et al. 2005, Gamlin et al. 2006, Cataño et al. 2008, Bernal-Hernández et al. 2014). En este trabajo, se observó una inhibición del $32 \%$ de la actividad AChE en los trabajadores de la comunidad de Santiago de Pochotitán y $33 \%$ en los trabajadores de Jesús María Corte, con respecto a datos obtenidos de una población mestiza de la entidad. De acuerdo con el comité científico de plaguicidas del ICOH (International Commission on Occupational Health), un porcentaje de inhibición del $30 \%$ de la actividad AChE está clasificado como segundo nivel de vigilancia y es necesario verificar las condiciones de trabajo de la población (De la Iglesia y Delgado 2000). Cuando se estratificó la actividad con respecto al género, se observó que el $51 \%$ de las mujeres y $60 \%$ de los hombres presentaron actividad AChE por debajo de la considerada como normal en poblaciones mexicanas.

Por otra parte, se ha descrito que la BuChE es más sensible a la exposición de compuestos anticolinesterásicos como los POF (Podolak y Panasiuk 1997, Jaga y Dharmani 2003, Rojas-García et al. 2011, Bernal-Hernández et al. 2014). Al respecto, Soltaninejad et al. (2007) reportaron una disminución en la actividad BuChE con ausencia de efecto sobre la $\mathrm{AChE}$ en individuos intoxicados con POF. Asimismo, Jintana et al. (2009), reportaron que el efecto de la exposición a POF sobre las colinesterasas fue más evidente sobre la actividad BuChE. En este estudio se observó que el $50 \%$ de las mujeres y $60 \%$ de los hombres presentaron valores por debajo de los considerados normales. Si bien se observó sintomatología asociada con la exposición a plaguicidas en este estudio, no se observó una inhibición de la AChE que comprometa la salud de los jornaleros. Estos resultados son similares a lo reportado por Nicole et al. (2003), quienes describen la presencia de síntomas como cefalea, irritabilidad, insomnio y debilidad, sin alteración en la actividad de las colinesterasas.

\section{CONCLUSIÓN}

La población de estudio se encuentra expuesta de manera ocupacional y crónica a plaguicidas que están considerados como "extremadamente peligrosos" y no utiliza equipo de protección adecuado, lo que sin duda los pone en una situación de alta vulnerabilidad. Los datos publicados en este estudio 
permitirán contribuir al desarrollo de propuestas encaminadas a conocer el impacto a la salud por la exposición a plaguicidas, así como implementar estrategias de educación ambiental y comunicación de riesgo, que consideren los aspectos socioculturales de estas poblaciones en la exposición a estos compuestos.

\section{AGRADECIMIENTOS}

Los autores agradecen a la Red Temática de Toxicología de Plaguicidas (CONACyT-280045) y al Fondo SS/IMSS/ISSSTE-CONACyT-262284. Así como, a la Comisión Nacional para el Desarrollo de los Pueblos Indígenas (CDI) Nayarit, específicamente al Ing. Efraín Moreno Hernández director del Centro Coordinador para el Desarrollo Indígena de Tepic y a su grupo de trabajo, por las facilidades otorgadas para las visitas a las comunidades de estudio..

\section{REFERENCIAS}

Álvarez I. (2015). La lucha territorial de los huicholes. Reconocimiento político e integridad cultural en un mismo enunciado. Ulúa 25, 123-142.

Bernal-Hernández Y.Y., Medina I.M., Barrón B.S., Robledo M.L., Girón M.I., Pérez N.E., Quintanilla B., Cerda R. y Rojas A.E. (2014). Paraoxonase 1 and its relationship with pesticide biomarkers in indigenous Mexican farmworkers. JOEM. 56 (3), 281-290pp. DOI: 10.1097/01.jom.0000438381.25597.88

Carmen A. y Waghiyi V. (2012). Indigenous women and environmental violence. A rights-based approach addressing impacts of environmental contamination on indigenous women, girls and future generations. Memorias. Primer simposio internacional de salud reproductiva y ambiental en mujeres indígenas. New York, U.S.A. 18 al 20 de enero, 2012. Web.

Cataño H.C., Carranza E., Huamani C. y Hernández A.F. (2008). Plasma cholinesterase levels and health symptoms in Peruvian Farm workers exposed to organophosphate pesticides. Arch. Environ. Contam. Toxicol. 55 (1), 153-159.

DOI: $10.1007 / \mathrm{s} 00244-007-9095-0$

CDI (2000). Regiones indígenas de México. Comisión Nacional para el Desarrollo de los Pueblos Indígenas. Ciudad de México, México.146pp.

Costa L.G., Vitalone A., Cole T.B. y Fulong C.E. (2005). Modulation of paraoxonase (PON1) activity. Biochem. Pharmacol. 69 (4), 541-550.

DOI: $10.1016 /$ j.bcp.2004.08.027
De la Iglesia A. y Delgado P. (2000) Plaguicidas: Neurotoxicidad y vigilancia de la salud. Revista del Instituto Nacional de Seguridad e Higiene en el Trabajo. 8, 4-14.

Díaz P. y Salinas S. (1998). Una Cultura Envenenada: El Caso de los Campesinos Indígenas Huichol. Noticias de Abya Yala. 11 (1), 10-12.

Díaz P. y Salinas S. (2002). Plaguicidas, tabaco y salud: el caso de los jornaleros huicholes, jornaleros mestizos y ejidatarios en Nayarit, México. PGO, Oaxaca, México. 265 pp.

Eckersley R. M. (2007). Culture, spirituality, religion and health: looking at the big picture. Med J Aust. 186 (10), 54-56. DOI:10.1.1.184.187

FAO (2003). Código Internacional de conducta para la distribución y utilización de plaguicidas. Organización de las Naciones Unidas para la agricultura y la alimentación. Código. Roma, Italia, 7 pp.

Gamlin J., Diaz P. y Hesketh T. (2006). Exposure of young children working on Mexican Tobacco plantations to organophosphorous and carbamic pesticides, indicated by cholinesterase depression. Child Care Health Dev. 33 (3), 246-248.

DOI: $10.1111 / \mathrm{j} .1365-2214.2006 .00702 . x$

Gamlin J.B. (2013). Pesticides and maternal child health, experience and the construction of knowledge among the Huichol. Tesis de doctorado. Institute of Global Health. University College London. Londres, Reino Unido, 366 pp.

Gamlin J. (2016). Huichol migrant laborers and pesticides: structural violence and cultural confounders. Med. Anthropol. Q. 30 (3), 303-320. DOI: 10.1111/maq. 12249

Jaga K. y Dharmani C. (2003). Sources of exposure to and public health implications of organophosphate pesticides. Rev. Panam. Salud Pública 14 (3), 171-185.

Hernández A.F., López O., Rodrigo L., Gil F., Pena G., Serrano J.L., Parrón T., Álvarez J.C., Lorente J.A. y Pla A. (2005). Changes in erythrocyte enzymes in humans long-term exposed to pesticides: influence of several markers of individual susceptibility. Toxicol. Lett. 159 (1), 13-21.

DOI: $10.1016 /$ j.toxlet.2005.04.008

INEE (2006). Panorama educativo de México 2005 indicadores del sistema educativo nacional. Instituto Nacional para la Evaluación de la Educación. Anuario. Ciudad de México, México, 63pp.

INEGI (2010). Censo de población y vivienda 2010. [En línea]. http://www.inegi.org.mx/est/contenidos/ proyectos/ccpv/cpv2010/ 26/01/2017

INEGI (2016). Encuesta Intercensal 2015 [En línea]. http://www.beta.inegi.org.mx/proyectos/enchogares/ especiales/intercensal/ 10/12/2016

Jintana S., Sming K., Krongtong Y. y Thanyachai S. (2009). Cholinesterase Activity, Pesticide Exposure 
and Health Impact in a population exposed to organophosphates. Int. Arch. Occ. Env. Hea. 82 (7), 833-842. DOI: $10.1007 / \mathrm{s} 00420-009-0422-9$

Koenig H.G. (2007). Religion, spirituality and medicine in Australia: research and clinical practice. Med. J. Aust. 186 (10), 45-46.

Mackinlay H. (2008). Jornaleros agrícolas y agroquímicos en la producción del tabaco en Nayarit. Alteridades 18 (36), 123-143.

Navarrete F. (2008). Los pueblos indígenas de México, Comisión Nacional para el Desarrollo de los pueblos indígenas. Ciudad de México, México, 141pp.

Neupane D., Jørs E. y Brandt L. (2014). Pesticide use, erythrocyte acetylcholinesterase level and self-reported acute intoxication symptoms among vegetable farmers in Nepal: a cross-sectional study. Environ. Health 13, 2-7. DOI: $10.1186 / 1476-069 X-13-98$

Neurath J. (2003). Huicholes pueblos indígenas del México contemporáneo. Comisión Nacional para el desarrollo de los pueblos indígenas. Ciudad de México, México. 31pp.

Nicole Y., Lockridge O., Masson P., Fontecilla J.C. y Nachon F. (2003). Crystal Structure of Human Butyrylcholinesterase and of Its Complexes with Substrate and Products. J. Biol. Chem. 278 (42), 41141-41147. DOI: $10.1074 / j b c . M 210241200$

Ochoa H. (2001). La organización territorial huichol. Tesis de licenciatura. Departamento de geografía y organización territorial. Centro Universitario de Ciencias Sociales y Humanidades. Universidad de Guadalajara. Guadalajara, Jalisco, México, 129 pp.

OMS (1999). Informe de la consulta internacional sobre la salud de los pueblos indígenas. Organización Mundial de la Salud. Informe. Ginebra, Suiza, 60 pp.
OMS. (2009). The WHO Recommended Classification of Pesticides by Hazard and Guidelines to Classification. Guía, Stuttgart, Alemania, 78 pp.

Pacheco L.C. (1999). Nomás venimos a malcomer jornaleros indios en el tabaco en Nayarit. Universidad Autónoma de Nayarit, Tepic, México. 309 pp.

Podolak M. y Panasiuk L. (1997). Biological indicators for the assessment of human exposure to organophosphorus compounds. Przegl. Lek. 54 (10), 719-722.

Ramos J.A. (2012). Propuesta de dinamización turística de la comunidad Zapote de Picachos, Municipio de Tepic, Nayarit, a través de un proyecto etnoturístico. Tesis de maestría. Unidad Académica de Turismo, Universidad Autónoma de Nayarit. Tepic, México, 113 pp.

Rojas-García A., Medina I., Robledo M., Barrón B., Girón M., Velázquez J., González C., Albores A., Quintanilla B., Ostrosky P., Rojas M., Pérez N. y López J. (2011). Hematological, biochemical effects and self-reported symptoms in pesticides retailers. J. Occup. Environ. Med. 53 (5), 517-521.

DOI: $10.1097 / J O M .0 b 013 \mathrm{e} 318215 \mathrm{fbf} 2$

Salinas S y Díaz P. (2000). Taller de capacitación sobre riesgos en el uso de plaguicidas. Carta descriptiva. [En línea]. http://hypinfo.org/documentos/taller_dh.pdf 04/01/2017

Soltaninejad K., Shadnia S., Afkhami M., Saljooghi R., Mohammadirad A. y Abdollahi M. (2007). Blood $\beta$-glucuronidase as a Suitable Biomarker at Acute Exposure of Severe Organophosphorus Poisoning in Human. Hum. Exp. Toxicol. 26 (12), 963-966. DOI: $10.1097 / \mathrm{JOM} .0 \mathrm{~b} 013 \mathrm{e} 318215 \mathrm{fbf} 2$ 